\title{
Meta-analysis of chest compression-only versus conventional cardiopulmonary resuscitation by bystanders for adult with out-of-hospital cardiac arrest
}

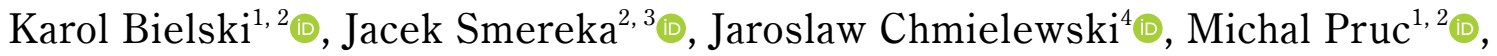
Francesco Chirico $^{5,6}{ }^{\circ}$, Aleksandra Gasecka ${ }^{7,8}{ }^{\infty}$, Nataliia Litvinova ${ }^{9}$, Milosz J. Jaguszewski ${ }^{10} \odot$, Grazyna Nowak-Starz ${ }^{11}\left({ }^{1}\right.$, Zubaid Rafique $^{12}$, , Frank W. Peacock ${ }^{12}$, Lukasz Szarpak ${ }^{2,13,14}$

${ }^{1}$ Institute of Outcomes Research, Polonia Academy, Czestochowa, Poland; ${ }^{2}$ Polish Society of Disaster Medicine, Warsaw, Poland; ${ }^{3}$ Department of Emergency Medical Service, Medical University of Wroclaw,

Poland; ${ }^{4}$ College of Rehabiliation, Warsaw, Poland; ${ }^{5}$ Postgraduate School of Occupational Medicine, Università Cattolica del Sacro Cuore, Rome, Italy; ${ }^{6} \mathrm{Health}$ Service Department, State Police, Ministry of Interior, Milan, Italy; ${ }^{7}$ Laboratory of Experimental Clinical Chemistry, Amsterdam University Medical Center, Amsterdam, the Netherlands; ${ }^{8} 1^{\text {st }}$ Chair and Department of Cardiology, Medical University of Warsaw, Poland; ${ }^{9}$ European Medical School, International European University, Kiev, Ukraine;

${ }^{10} 1^{\text {st }}$ Department of Cardiology, Medical University of Gdansk, Poland; ${ }^{11}$ Collegium Medicum Institute of Health Sciences, Kochanowski University, Kielce, Poland; ${ }^{12}$ Henry JN Taub Department of Emergency Medicine, Baylor College of Medicine Houston, TX, United States; ${ }^{13}$ Maria Sklodowska-Curie Medical Academy, Warsaw, Poland; ${ }^{14}$ Maria Sklodowska-Curie Bialystok Oncology Center, Bialystok, Poland

\section{This paper was guest edited by Prof. Togay Evrin}

\begin{abstract}
Background: According to the guidelines of cardiopulmonary resuscitation (CPR) conducted by bystanders, two methods of $C P R$ are feasible: standard CPR (sCPR) with mouth-to-mouth ventilations and continuous chest compression-only CPR (CCC) without rescue breathing. The goal herein, was to evaluate the effect of $s C P R$ (30:2) and CCC on resuscitation outcomes in patients with out-of-hospital cardiac arrest (OHCA) patients.

Methods: This study was a systematic review and meta-analysis. Using standardized criteria, PubMed, Web of Science, Scopus, EMBASE and Cochrane Collaboration were searched for trials assessing the effect of $s C P R$ vs. CCC on resuscitation outcomes after adult OHCA. Random-effects model meta-analysis was applied to calculate the mean deviation (MD), odds ratio (OR) and $95 \%$ confidence interval (CI).

Results: Overall, 3 randomized controlled trials and 12 non-randomized trials met the inclusion criteria. Survival to hospital discharge with $s C P R$ was $10.2 \%$ compared to $9.3 \%$ in the CCC group $(O R=1.04 ; 95 \%$ CI: 0.93-1.16; $p=0.46)$. Survival to hospital discharge with good neurological outcome measured with the cerebral performance category (CPC 1 or 2) was $6.5 \%$ for $s C P R$ vs. $5.8 \%$ for CCC $(O R=1.00 ; 95 \%$ CI: 0.84-1.20; $p=0.98)$. Prehospital return of spontaneous circulation (ROSC) in
\end{abstract}

Address for correspondence: Lukasz Szarpak, Assoc. Prof. PhD, DPH, MBA, DBA, LL.M, Maria Sklodowska-Curie Medical Academy, ul. Solidarności 12, 03-411 Warszawa, Poland, tel: +48 500186225, e-mail: lukasz.szarpak@gmail.com 
$s C P R$ and CCC groups was $15.9 \%$ and $14.8 \%$, respectively $(O R=1.13 ; 95 \%$ CI: $0.91-1.39 ; p=0.26)$. Survival to hospital admission with ROSC occurred in $29.5 \%$ of the $s C P R$ group compared to $28.4 \%$ in CCC group (OR = 1.20; 95\% CI: 0.89-1.63; $p=0.24)$.

Conclusions: This systematic review and meta-analysis concluded that there were no significant differences in the resuscitation outcomes between the use of standard cardiopulmonary resuscitation and chest compression only. (Cardiol J)

Key words: out-of-hospital cardiac arrest, cardiopulmonary resuscitation, chest compression, continuous compressions

\section{Introduction}

Despite significant advances in the delivery of care, the survival rate of out-of-hospital cardiac arrest (OHCA) is poor [1]. According to study by Nadolny et al. [2] return of spontaneous circulation refers to $35.1 \%$ OHCA patients and only $28.7 \%$ patients are admitted to the hospital. Current recommendations of the American Heart Association (AHA) [3], as well as the European Resuscitation Council (ERC) [4], place great emphasis on high-quality cardiopulmonary resuscitation (CPR). This includes high-quality chest compressions [5] and minimizing interruptions during chest compressions [6].

Cardiopulmonary resuscitation can be a heavy burden for bystanders. In the past, bystanders often did not undertake CPR due to the resistance associated with the need to perform mouth-to-mouth ventilation $[7,8]$. For this reason, the ERC and AHA guidelines have introduced two possible CPR techniques for bystanders. The first is the standard method of performing cycles based on 30 compressions with a pause for two ventilations $(30: 2)$. The second is based on continuous chest compression without the need for pauses for rescue breaths - which is intended to encourage people to undertake more frequent resuscitation efforts [9].

The systematic review and meta-analysis are aimed to evaluate the effect of standard CPR (sCPR) (30:2) and continuous chest compressions without rescue breaths (CCC) on resuscitation outcomes in patients with OHCA.

\section{Methods}

This systematic review and meta-analysis were conducted in accordance with the Cochrane Handbook for Systematic Reviews of Interventions [10] and the Preferred Reporting Items for Systematic Reviews and Meta-Analyses (PRISMA) guidelines [11].

\section{Search strategy}

Screening of papers and the data extraction were undertaken by two independent authors (K.B. and M.P.), using predefined selection criteria and a data extraction sheet. Disagreements were resolved by a third investigator (L.S.). PubMed, Web of Science, Scopus, EMBASE and Cochrane Collaboration database, English language articles published from the databases inception to $21^{\text {st }}$ July 2021 were searched. The search was performed using the following terms: "cardiopulmonary arrest" OR "heart arrest" OR "cardiac arrest” OR "heart attack" OR "sudden cardiac death" OR "out-oh-hospital cardiac arrest” OR “OHCA" OR “asystole” OR "PEA" OR "pulseless electrical activity" OR "VF" OR "ventricular fibrillation" OR "VT" OR "ventricular tachycardia” AND "resuscitation” OR "CPR” OR "chest compression" OR "30:2" OR "conventional resuscitation” OR "continuous compression”. Additionally, we reviewed the bibliographies of the identified trials and evaluated review articles for relevant references.

\section{Study selection}

Included studies were required to document the following parameters: (1) Participants; OHCA in adult patients, (2) Intervention; conventional cardiopulmonary resuscitation, (3) Comparison; chest compression without ventilation (CC-CPR), (4) Outcomes; detailed information for mortality, (5) Study design; randomized controlled trials and observational studies.

Studies were excluded if they met the following criteria: (A) studies including pediatric patient; (B) were classed as Letter to Editor, Correspondence, or as an Editorial; (C) animal or simulation trials; (D) conference abstract; (E) guidelines. Studies were also excluded if the full paper was not available in English. 


\section{Outcomes}

Primary end points were in-hospital or 30-day mortality and survival to hospital discharge with good neurological outcome defined as the cerebral performance category (CPC) score 1 or 2 [12]. Secondary end points were return of spontaneous circulation (ROSC) and survival to hospital admission.

\section{Data extraction and quality assessment}

Two reviewers (K.B. and J.C.) independently extracted and entered the following data into a predefined extraction table: study characteristics, mortality, and neurologic outcome. If multiple publications of the same dataset were obvious or confirmed by the authors, the one with the most extractable and complete information was chosen. Any discrepancies were resolved through discussion with a third reviewer (L.S.).

The risk of bias (RoB) of the included studies was independently assessed by 3 reviewers (K.B., A.G. and J.S.) according to the revised tool for risk of bias in randomized trials (RoB 2 tool) [13] and Risk of Bias in Non-randomized Studies - of Interventions (ROBINS-I) [14]. All disagreements were resolved by referral to a third author (L.S.) if necessary. ROBINS examines seven domains of bias: (1) confounding; (2) selection of participants; (3) classification of interventions; (4) deviations from intended interventions; (5) missing data; (6) measurement of outcomes; and (7) selection of the reported result. The overall ROBINS-I judgment at domain and study level was attributed according to the criteria specified in the ROBVIS tool [15].

\section{Statistical analysis}

All statistical analysis were performed using the STATA software (Version 13.0 StataCorp) and the Review Manager software (RevMan version 5.4; The Cochrane Collaboration 2014). Randomeffects meta-analyses of continuous data with mean deviations (MDs) and their 95\% confidence intervals (95\% CIs) [16] were performed. For dichotomous data, odds ratios (ORs) as the effect measure with $95 \%$ CI were used. When the continuous outcome was reported in a study as median, range, and interquartile range, means and standard deviations were estimated using the formula described by Hozo et al. [17]. For meta-analysis the random effects model was used (assuming a distribution of effects across studies) to weigh estimates of studies in proportion to their significance [10].

Heterogeneity was assessed by the Cochran's $\mathrm{Q}$ test and $\mathrm{I}^{2}$ statistics, with low, moderate, and high levels of heterogeneity designated as $25 \%, 50 \%$, and $75 \%$, respectively [18]. All variables were analyzed using the DerSimonian-Laird random effects model. Where there were fewer than 10 included studies, publication bias was unable to be formally assessed [10]. A p-value of less than 0.05 (2-tailed) was considered statistically significant.

\section{Results}

\section{Search results and study characteristics}

A PRISMA flowchart, including the reasons for excluding studies, is shown in Figure 1. A total of 1319 records were identified, of which duplicate records and further 783 records were excluded based on the title and abstract evaluation. After review of the remaining 39 articles in full, 15 articles [19-33] ultimately met the inclusive criteria and were included in the meta-analysis.

Table 1 details the characteristics of the selected trials. Included trials were published between 2000 and 2021, totaling 220,945 OHCA patients (80,051 in standard CPR group and 140,894 in the CCC group). Overall, 3 studies were randomized controlled trials [19-21] with the remaining being non-randomized [22-33].

\section{Risk of bias in included studies}

RoB 2 and ROBINS-I tools were used to evaluate methodological quality and risk of bias respectively for the randomized and non-randomized studies. Summary of the risk of bias of included trials is presented in Supplementary data (Suppl. Figs. S1, S2).

\section{Meta-analysis outcomes}

A polled analysis of the 13 studies indicated survival to hospital discharge with SCPR was $10.2 \%$ compared to the $9.3 \%$ in CCC group (OR = 1.04; 95\% CI: 0.93-1.16; $\mathrm{p}=0.46$; Table 2). Sub-analysis comparing survival to hospital discharge between sCPR and CCC was not significantly different in randomized ( $6.2 \%$ vs. $6.1 \%$, respectively; $\mathrm{OR}=0.94$; 95\% CI: $0.78-1.12 ; \mathrm{p}=0.48$ ) or non-randomized trials $(10.9 \%$ vs. $9.8 \%$; OR $=1.08$; $95 \%$ CI: $0.95-1.24$; $\mathrm{p}=0.24)$.

Survival to hospital discharge with good neurological outcome (CPC 1 or 2) was reported in 7 studies and was $6.5 \%$ for sCPR compared to $5.8 \%$ for CCC $(\mathrm{OR}=1.00$; 95\% CI: $0.84-1.20$; $\mathrm{p}=0.98$ ). Five studies reported ROSC. Polled analysis showed that ROSC in SCPR and CCC groups was $15.9 \%$ and $14.8 \%$, respectively $(\mathrm{OR}=1.13$; 95\% CI: 0.91-1.39; $\mathrm{p}=0.26$ ). 


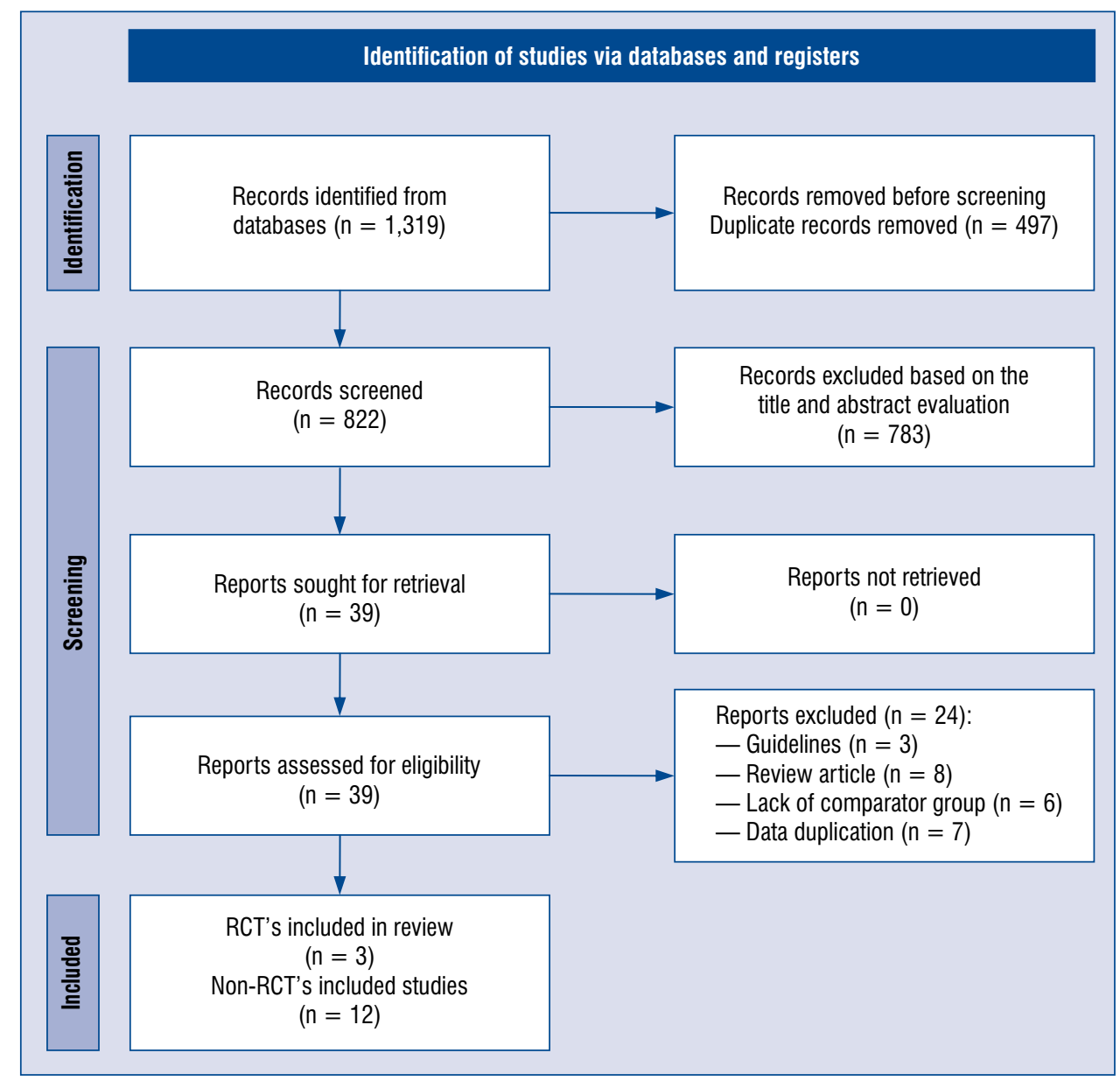

Figure 1. Flow diagram showing stages of database searches and study selection as per Preferred Reporting Items for Systematic reviews and Meta-Analysis (PRISMA) guideline; RCT — randomized controlled trials.

Survival to hospital admission after ROSC was observed in $29.5 \%$ of participants in the SCPR group compared to $28.4 \%$ in CCC group $(\mathrm{OR}=1.20$; 95\% CI: $0.89-1.63 ; \mathrm{p}=0.24$ ).

\section{Discussion}

In this systematic review and meta-analysis, standard CPR with chest compression only for adult OHCA resuscitation was compared. No significant differences were found between both arms for all outcomes. It was felt that data supporting this important finding is sufficient to recommend changes in standard practice. While the number of individuals enrolled in the totality of randomized controlled trials [19-21] included in the present analysis is relatively limited ( $\mathrm{n}=3,737)$, not a single randomized trial demonstrated a significant clinical outcome benefit with the application of rescue breathing. When considered in conjunction with the large number of patients included in the observational trials $(n=213,123)$, the summation of the data equates to 216,680 patients and is sufficient to support the removal of rescue breathing from standard guidelines of bystander CPR in OHCA.

There are multiple reasons that compression only CPR should be the preferred option for bystander CPR. These include the fact that shared secretions that occur from mouth-to-mouth resuscitation serves as an impediment for adoption in unrelated bystanders, and because it is easier to instruct an unexperienced provider by telephone in the performance of chest compression only CPR when guidance is obtained remotely $[34,35]$. Furthermore, in the time of a global pandemic, the performance of rescue breathing must be considered an avoidable high-risk activity for the transmission of pathogens from the patient to the rescue breathing provider [36-39]. 


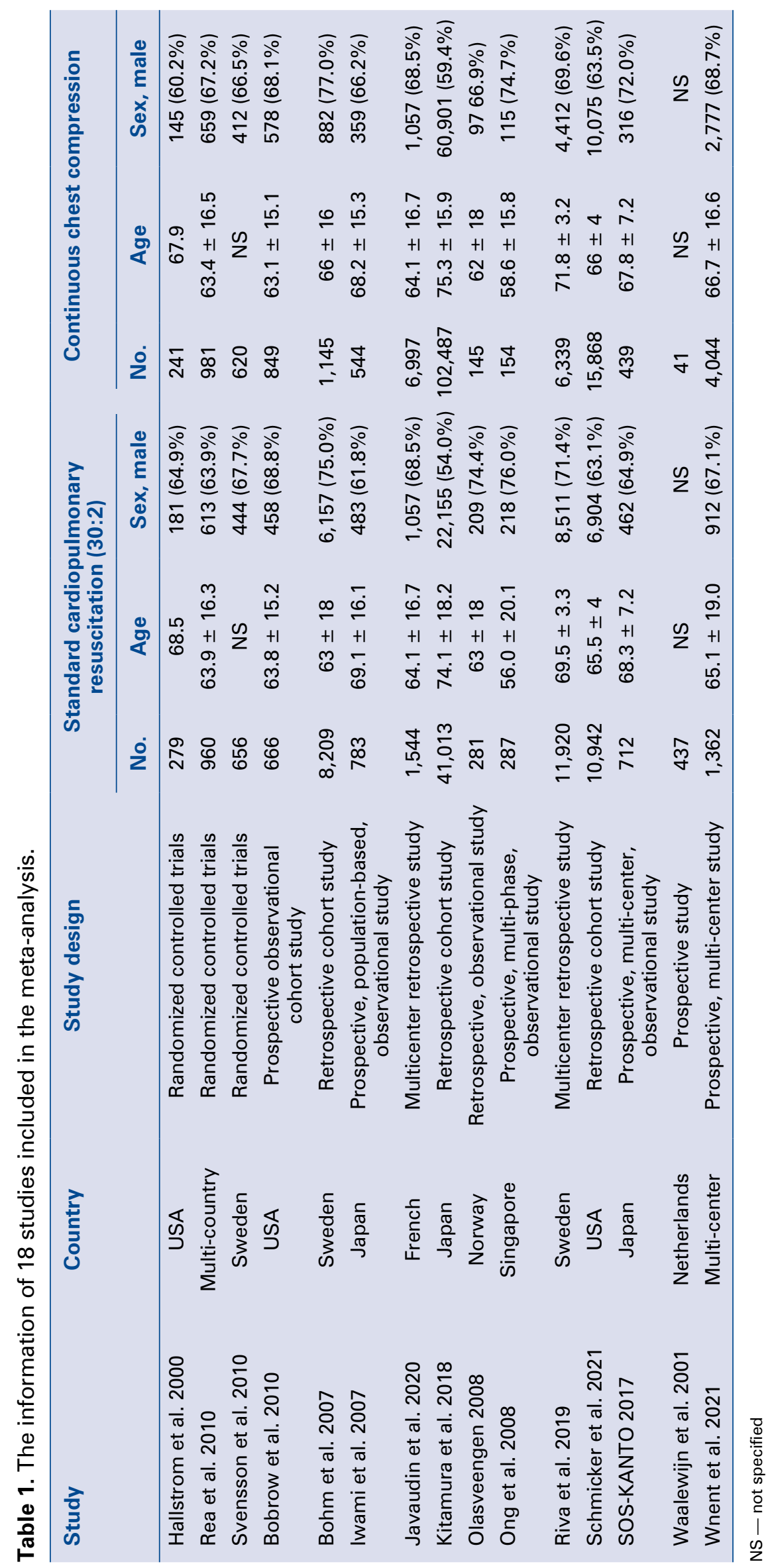




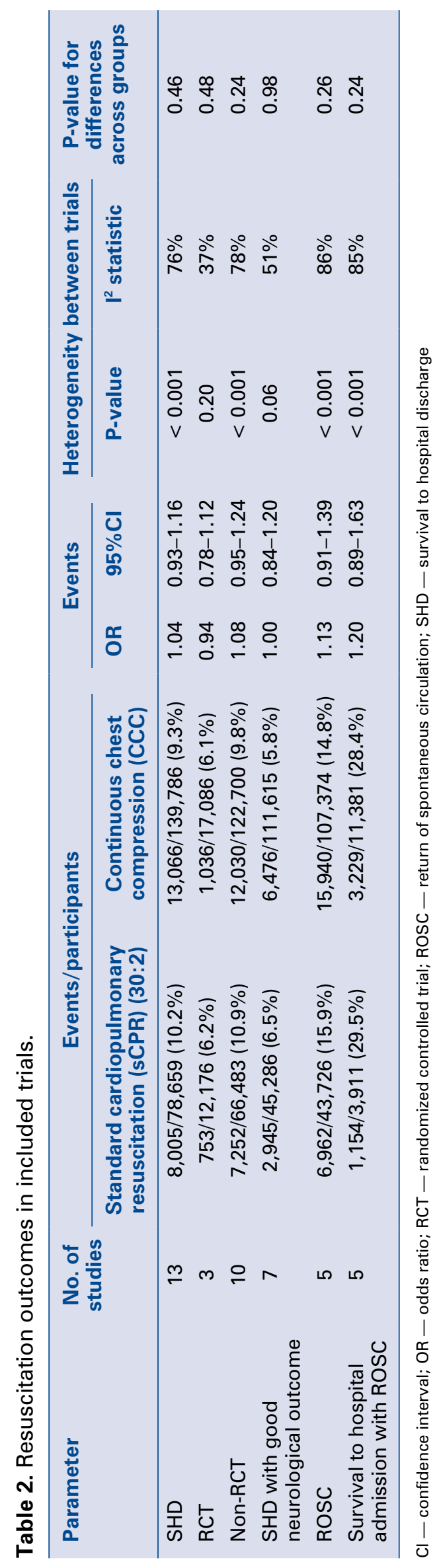

It should be noted that the findings of the randomized controlled trials most likely represent the "best case scenario". This is because the performance of these trials occurred in environments with extremely well-developed Emergency Medical Service (EMS) systems; most likely some of the most sophisticated on the globe. The fact that their outcomes show no benefit with the addition of rescue breathing to standard CPR practice suggests that there would be even less outcome improvement in systems with longer times to advanced cardiac life support and transport to hospital. Further, the majority of patients enrolled in the randomized controlled trials occurred in urban environments, in areas of relative wealth. It would not be expected that the addition of rescue breathing would be improved in a rural or poor environment.

Finally, while the numeric majority of the present data is obtained from observational trials performed in industrialized nations with relatively high performing EMS infrastructure, it was found that the summation of their reported outcomes were similar to the randomized controlled trials. In the largest $(n=143,500)$ observational trial [26], multivariate analysis and propensity matching reported significant outcome improvements with chest compression only CPR. Considering the next 3 largest observational studies [23, 29, 30], $\mathrm{n}=$ $=68,530$, found mixed results, with both Riva et al. [29], $\mathrm{n}=30,445$, and Schmicker et al. [30], $\mathrm{n}=$ $=26,810$, reporting improvements by the addition of rescue breathing, and Bohm et al. [23], $\mathrm{n}=11,275$, no difference in outcomes was found.

This meta-analysis should be interpreted with consideration of certain limitations. First, only 3 of the included studies are randomized controlled trials. The others are non-randomized studies that are assumed to carry a higher risk of unmeasured bias than randomized controlled trials. Another limitation is the fact that the included studies limited outcomes to discharge from hospital or 30 days after cardiac arrest. Only one study by Iwami et al. [24] reported an annual survival rate of $5.5 \%$ for standard CPR and $5.0 \%$ for CC-CPR, respectively.

\section{Conclusions}

In conclusion, the systematic review and metaanalysis concluded that there were no significant differences in the resuscitation outcomes between the use of standard CPR and chest compression only. The choice of standard CPR and chest compression without mouth-to-mouth ventilation 
remains the bystander's preference, however guideline changes may be considered.

\section{Acknowledgments}

The study was supported by the ERC Research Net and by the Polish Society of Disaster Medicine.

\section{Conflict of interest: None declared}

\section{References}

1. Borkowska MJ, Smereka J, Safiejko K, et al. Out-of-hospital cardiac arrest treated by emergency medical service teams during COVID-19 pandemic: a retrospective cohort study. Cardiol J. 2021; 28(1): 15-22, doi: 10.5603/CJ.a2020.0135, indexed in Pubmed: 33140396 .

2. Nadolny K, Bujak K, Kucap M, et al. The Silesian Registry of Out-of-Hospital Cardiac Arrest: Study design and results of a three-month pilot study. Cardiol J. 2020; 27(5): 566-574, doi: 10.5603/CJ.a2018.0140, indexed in Pubmed: 30444257.

3. Panchal AR, Bartos JA, Cabañas JG, et al. Part 3: Adult Basic and Advanced Life Support: 2020 American Heart Association Guidelines for Cardiopulmonary Resuscitation and Emergency Cardiovascular Care. Circulation. 2020; 142(16_suppl_2): S366-S468, doi: 10.1161/CIR.0000000000000916, indexed in Pubmed: 33081529 .

4. Olasveengen TM, Semeraro F, Ristagno G, et al. European Resuscitation Council Guidelines 2021: Basic Life Support. Resuscitation. 2021; 161: 98-114, doi: 10.1016/j.resuscitation.2021.02.009, indexed in Pubmed: 33773835.

5. Smereka J, Madziala M, Szarpak L. Comparison of two infant chest compression techniques during simulated newborn cardiopulmonary resuscitation performed by a single rescuer: a randomized, crossover multicenter trial. Cardiol J. 2019; 26(6): 761-768, doi: 10.5603/CJ.a2018.0090, indexed in Pubmed: 30155866.

6. Ewy GA, Zuercher M, Hilwig RW, et al. Improved neurological outcome with continuous chest compressions compared with 30:2 compressions-to-ventilations cardiopulmonary resuscitation in a realistic swine model of out-of-hospital cardiac arrest. Circulation. 2007; 116(22): 2525-2530, doi: 10.1161/CIRCULATIONAHA.107.711820, indexed in Pubmed: 17998457.

7. Al-Jeabory M, Safiejko K, Bialka S, et al. Impact of COVID-19 on bystander cardiopulmonary resuscitation in out-of-hospital cardiac arrest: Is it as bad as we think? Cardiol J. 2020; 27(6): 884-885, doi: 10.5603/CJ.a2020.0179, indexed in Pubmed: 33346369.

8. Jorge-Soto C, Abilleira-González M, Otero-Agra M, et al. Schoolteachers as candidates to be basic life support trainers: a simulation trial. Cardiol J. 2019; 26(5): 536-542, doi: 10.5603/ CJ.a2018.0073, indexed in Pubmed: 30009374.

9. Majer J, Jaguszewski MJ, Frass M, et al. Does the use of cardiopulmonary resuscitation feedback devices improve the quality of chest compressions performed by doctors? A prospective, randomized, cross-over simulation study. Cardiol J. 2019; 26(5): 529-535, doi: 10.5603/CJ.a2018.0091, indexed in Pubmed: 30155865.

10. Higgins JP, Thomas J, Chandler J, Cumpston M, Li T, Page MJ, Welch VA, eds. Cochrane Handbook for Systematic Reviews of Interventions version 6.0 (updated July 2019); 2019.

11. Page MJ, McKenzie JE, Bossuyt PM, et al. Updating guidance for reporting systematic reviews: development of the PRISMA 2020 statement. J Clin Epidemiol. 2021; 134(3): 103-112, doi: 10.1016/j.jclinepi.2021.02.003, indexed in Pubmed: 33577987.
12. Reynolds EC, Zenasni Z, Harrison DA, et al. How do information sources influence the reported Cerebral Performance Category (CPC) for in-hospital cardiac arrest survivors? An observational study from the UK National Cardiac Arrest Audit (NCAA). Resuscitation. 2019; 141: 19-23, doi: 10.1016/j.resuscitation.2019.05.035, indexed in Pubmed: 31199943.

13. Sterne JAC, Savović J, Page MJ, et al. RoB 2: a revised tool for assessing risk of bias in randomised trials. BMJ. 2019; 366: 14898, doi: 10.1136/bmj.14898, indexed in Pubmed: 31462531.

14. Sterne JAC, Hernán MA, Reeves BC, et al. ROBINS-I: a tool for assessing risk of bias in non-randomised studies of interventions. BMJ. 2016; 355: i4919, doi: 10.1136/bmj.i4919, indexed in Pubmed: 27733354.

15. McGuinness LA, Higgins JPT. Risk-of-bias VISualization (robvis): An $\mathrm{R}$ package and Shiny web app for visualizing risk-ofbias assessments. Res Synth Methods. 2021; 12(1): 55-61, doi: 10.1002/jrsm.1411, indexed in Pubmed: 32336025.

16. DerSimonian R, Laird N, DerSimonian R, et al. Meta-analysis in clinical trials. Control Clin Trials. 1986; 7(3): 177-188, doi: 10.1016/0197-2456(86)90046-2, indexed in Pubmed: 3802833.

17. Hozo SP, Djulbegovic B, Hozo I. Estimating the mean and variance from the median, range, and the size of a sample. BMC Med Res Methodol. 2005; 5: 13, doi: 10.1186/1471-2288-5-13, indexed in Pubmed: 15840177.

18. Higgins JPT, Thompson SG, Deeks JJ, et al. Measuring inconsistency in meta-analyses. BMJ. 2003; 327(7414): 557-560, doi: 10.1136/bmj.327.7414.557, indexed in Pubmed: 12958120.

19. Hallstrom A, Cobb L, Johnson E, et al. Cardiopulmonary resuscitation by chest compression alone or with mouth-to-mouth ventilation. N Engl J Med. 2000; 342(21): 1546-1553, doi: 10.1056/ NEJM200005253422101, indexed in Pubmed: 10824072.

20. Rea T, Fahrenbruch C, Culley L, et al. CPR with chest compression alone or with rescue breathing. N Engl J Med. 2010; 363(5): 423-433, doi: 10.1056/nejmoa0908993.

21. Svensson L, Bohm K, Castrèn M, et al. Compression-only CPR or standard CPR in out-of-hospital cardiac arrest. N Engl J Med. 2010; 363(5): 434-442, doi: 10.1056/nejmoa0908991.

22. Panchal AR, Bobrow BJ, Spaite DW, et al. Chest compression-only CPR by lay rescuers and survival from out-of-hospital cardiac arrest. JAMA. 2010; 304(13): 1447-1454, doi: 10.1001/ jama.2010.1392, indexed in Pubmed: 20924010.

23. Bohm K, Rosenqvist M, Herlitz J, et al. Survival is similar after standard treatment and chest compression only in out-of-hospital bystander cardiopulmonary resuscitation. Circulation. 2007; 116(25): 2908-2912, doi: 10.1161/CIRCULATIONAHA.107.710194, indexed in Pubmed: 18071077.

24. Iwami T, Kawamura T, Hiraide A, et al. Effectiveness of bystander-initiated cardiac-only resuscitation for patients with out-ofhospital cardiac arrest. Circulation. 2007; 116(25): 2900-2907, doi: 10.1161/CIRCULATIONAHA.107.723411, indexed in Pubmed: 18071072 .

25. Javaudin F, Raiffort J, Desce N, et al. GR-RéAC. Neurological outcome of chest compression-only bystander CPR in asphyxial and non-asphyxial out-of-hospital cardiac arrest: an observational study. Prehosp Emerg Care. 2020 [Epub ahead of print]: 1-25, doi: 10.1080/10903127.2020.1852354, indexed in Pubmed: 33205692.

26. Kitamura T, Kiyohara K, Nishiyama C, et al. Chest compression-only versus conventional cardiopulmonary resuscitation for bystander-witnessed out-of-hospital cardiac arrest of medical origin: A propensity score-matched cohort from 143,500 patients. Resuscitation. 2018; 126: 29-35, doi: 10.1016/j.resuscitation.2018.02.017, indexed in Pubmed: 29476890. 


\section{Cardiology Journal}

27. Olasveengen TM, Wik L, Steen PA. Standard basic life support vs. continuous chest compressions only in out-of-hospital cardiac arrest. Acta Anaesthesiol Scand. 2008; 52(7): 914-919, doi: 10.1111/j.1399-6576.2008.01723.x, indexed in Pubmed: 18702753.

28. Ong ME, Ng FS, Anushia P, et al. Comparison of chest compression only and standard cardiopulmonary resuscitation for out-of-hospital cardiac arrest in Singapore. Resuscitation. 2008; 78(2): 119-126, doi: 10.1016/j.resuscitation.2008.03.012, indexed in Pubmed: 18502559.

29. Riva G, Ringh M, Jonsson M, et al. Survival in out-of-hospital cardiac arrest after standard cardiopulmonary resuscitation or chest compressions only before arrival of emergency medical services: nationwide study during three guideline periods. Circulation. 2019 [Epub ahead of print], doi: 10.1161/CIRCULATIONAHA.118.038179, indexed in Pubmed: 30929457.

30. Schmicker RH, Nichol G, Kudenchuk P, et al. CPR compression strategy 30:2 is difficult to adhere to, but has better survival than continuous chest compressions when done correctly. Resuscitation. 2021; 165: 31-37, doi: 10.1016/j.resuscitation.2021.05.027, indexed in Pubmed: 34098033.

31. SOS-KANTO study group. Cardiopulmonary resuscitation by bystanders with chest compression only (SOS-KANTO): an observational study. Lancet. 2007; 369(9565): 920-926, doi: 10.1016/ s0140-6736(07)60451-6.

32. Waalewijn RA, Tijssen JG, Koster RW. Bystander initiated actions in out-of-hospital cardiopulmonary resuscitation: results from the Amsterdam Resuscitation Study (ARRESUST). Resuscita- tion. 2001; 50(3): 273-279, doi: 10.1016/s0300-9572(01)00354-9, indexed in Pubmed: 11719156.

33. Wnent J, Tjelmeland I, Lefering $\mathrm{R}$, et al. To ventilate or not to ventilate during bystander CPR - A EuReCa TWO analysis. Resuscitation. 2021 [Epub ahead of print], doi: 10.1016/j.resuscitation.2021.06.006, indexed in Pubmed: 34146622.

34. Kłosiewicz T, Puślecki M, Zalewski R, et al. Analysis of the quality of chest compressions during resuscitation in an understaffed team - randomised crossover manikin study. Disaster Emerg Med J. 2020; 5(1): 24-29, doi: 10.5603/demj.a2020.0008.

35. Zalewski R, Przymuszala P, Klosiewicz T, et al. The effectiveness of 'practice while watching' technique for the first aid training of the chemical industry employees. Disaster Emerg Med J. 2019; 4(3): 83-91, doi: 10.5603/demj.2019.0018.

36. Szarpak L, Ruetzler K, Dabrowski M, et al. Dilemmas in resuscitation of COVID-19 patients based on current evidence. Cardiol J. 2020; 27(3): 327-328, doi: 10.5603/CJ.a2020.0066, indexed in Pubmed: 32419130.

37. Attila K, Ludwin K, Evrin T, et al. The impact of COVID-19 on airway management in prehospital resuscitation. Disaster Emerg Med J. 2020; 5(4): 216-217, doi: 10.5603/demj.a2020.0047.

38. Malysz M, Jaguszewski M, Szarpak L, et al. Comparison of different chest compression positions for use while wearing CBRN PPE: a randomized crossover simulation trial. Disaster Emerg Med J. 2020, doi: 10.5603/demj.a2020.0034.

39. Nolan JP, Monsieurs KG, Bossaert L, et al. European Resuscitation Council COVID-19 guidelines executive summary. Resuscitation. 2020; 153: 45-55, doi: 10.1016/j.resuscitation.2020.06.001, indexed in Pubmed: 32525022. 